Preprints of the

Max Planck Institute for

Research on Collective Goods

Bonn 2006/4

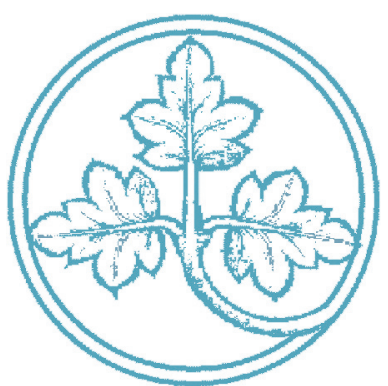

Noisy commitments:

The impact of information accuracy on efficiency

Eyal Ert / Andreas Nicklisch

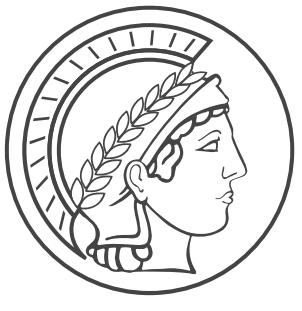




\section{Noisy commitments: The impact of information accuracy on efficiency}

Eyal Ert / Andraes Nicklisch

February 2006 


\title{
Noisy commitments: The impact of information accuracy on efficiency*
}

\author{
Eyal Ert ${ }^{\dagger} \&$ Andreas Nicklisch ${ }^{\ddagger}$
}

\begin{abstract}
We report an experiment designed to test the influence of noisy commitments on efficiency in a simple bargaining game. We investigate two different levels of commitment reliability in a variant of the peasant-dictator game. Theoretical analysis suggests that the reliability of commitments in this game does not affect efficiency. We find that accurate commitments promote efficiency, as expected by game theory. However, noisy commitments are found to impair efficiency. We explain this effect by the differences between incentives off the equilibrium path under conditions of accurate commitments and noisy commitments. This difference changes the game structure and in the current game facilitates more random responses.
\end{abstract}

Keywords: Commitments, efficiency, experimental economics, information, trust

[JEL] C7, C91, L15

${ }^{*}$ The authors would like to thank Felix Bierbrauer, Arndt Bröder, Christoph Engel, Ido Erev, Werner Güth, Felix Höffler, and Randolf Sloof for their support. This project was funded by the Minerva Foundation, Germany.

${ }^{\dagger}$ Max Wertheimer Minerva Center for Cognitive Research, Faculty of Industrial Engineering and Management, Israeli Institute of Technology, Haifa, Israel; email eyalert@techunix.technion.ac.il

${ }^{\ddagger}$ Max Planck Institute for Research on Collective Goods, Bonn, Germany; email nicklisch@coll.mpg.de 


\section{Trust and information}

Many economic interactions involve "trust games" (see Kreps, 1990; Berg et al., 1995). Whenever a hold-up problem (see Hart, 1995) is non-contractible, trading partners who have to make specific investments simply have to trust counterparts and run the risk that their counterparts will act opportunistically. There is a tension between the production or investment decision and the decision about how to share the gains of production. The former decision affects the efficiency of an interaction, whereas the latter one affects the distribution of gains among interaction partners. Unfortunately, distributional issues challenge efficiency issues. Economic efficiency is maximized when the players trust each other, but rational considerations are expected to lead traders to refrain from choosing the efficient investment. Recent research suggests that this problem can be addressed by providing information about the likely behavior of the different agents (see Lucking-Reiley et al., 1999; Burnham et al., 2000; Bolton et al., 2004). As demonstrated by van Huyck et al. (1995), this type of information is particularly effective when it involves early commitments. In a series of laboratory experiments, van Huyck et al. show for the peasant-dictator game that an early commitment indeed improves efficiency.

The main goal of the current research is to improve our understanding of the effect of early commitments on trust and efficiency. Specifically, we focus on the effect of noisy information about early commitments on investments and the implications for the efficiency of production. In the peasant-dictator game (van Huyck et al., 1995), a peasant endowed with beans can either consume them or plant them in a field, leading to gross rates greater than one. However, there is a dictator who taxes the peasant's harvest. A strategic analysis predicts that an early commitment will facilitate efficiency, and the party who is entitled to commit prior to the investment decision will receive all the surplus from the production. Yet, how does the noisy transmission of commitments affect the division of production gains? We address this question by studying a variant of the peasant-dictator game in which a dictator sends a message concerning the share of beans that he will leave for the peasant. This commitment, however, is not always accurate. It is possible that the true share of production committed to the peasant will be lower than the received signal about the commitment.

Our interest in the effect of noise on early commitments stems from two observations. First, many interesting examples of natural commitment devices are likely to be noisy. Consider, for an example, the sig-

nificant proportion of divorces after marriage. In many cases they occur because unforeseen events impair the parties' ability to fulfill their early 
commitments. Similarly, a restaurant that commits to a certain quality of food may not be able to provide the promised quality should its famous chef become ill. The accuracy of information may be also impaired by noisy transmissions, such as recommendations in tourist guides that might overestimate the quality of restaurants. Somehow, recommendations tend to bias information positively. ${ }^{1}$ One may also consider reputation systems for online sellers like those on eBay or Amazon. Most sellers have extraordinary reputation scores, but this says little about the accuracy of this recommendation. Therefore, in our analysis we stress the effect of - potentially - positively contaminated information on trade efficiency.

Second, whereas a theoretical analysis of our game suggests an equilibrium, irrespective of the level of noise, relevant behavioral studies can lead to contradicting predictions concerning the effect of noise on early commitments. One line of research demonstrates the importance of fairness in bargaining games (Rabin, 1993; Fehr \& Schmidt, 1999; Bolton \& Ockenfels, 2000). Yet, as mentioned earlier, distributional issues challenge efficiency issues. Thus, fairness concerns may cause efficiency losses. According to this line of research, for accurate commitments (i.e., commitment transmission which is not subject to noise) peasants refrain from efficient levels of investments when commitments violate their fairness concerns. Therefore, conflicting fairness norms lead to inefficiency, e.g., dictators in the peasant-dictator game may consider splitting the entire pie equally to be fair, while peasants expect an equal split of the production surplus. We show that noise reduces the number of conflicting fairness norms. Consequently, noise decreases the range of commitments causing inefficient investments. If commitments become noisy, efficiency is enhanced. Another line of research suggests that noise changes the structure of the game because it changes the incentives for behavior off the equilibrium path (Prasnikar \& Roth, 1992; Andreoni et al., 2002). This research implies that, because of such changes, noise will take players away from the equilibrium, towards a random choice. As a consequence, noise takes players away from the efficient equilibrium path and reduces efficiency. Hence contradictory predictions are derived from the existing literature.

The results of our laboratory experiments indicate that fairness concerns are indeed very important for players who have to commit themselves. This observation is not affected by the accuracy of information. However, the behavior of players who have to investment differs in ac-

\footnotetext{
${ }^{1} \mathrm{~A}$ restaurant that is underestimated by the writer of the tourist guide is not included in the guide at all. The reason is that typically the guide's purpose is to recommend high quality restaurants.
} 
cordance with the accuracy of the information. Pure selfish concerns direct investment decisions when commitments are accurately conveyed, whereas behavior is less structured when commitments are noisily conveyed. We show that this effect is caused by the different incentives off the equilibrium path for noisy commitment transmission such that noisy commitments impair efficiency.

This paper is organized as follows: Section 2 introduces the model of the peasant-dictator game with a commitment mechanism and develops the theoretical predictions for optimal behavior in this game. Section 3 elaborates hypotheses for the game in more detail and discusses counterarguments. Section 4 reports the experimental design and discusses the results of the laboratory experiments. Section 5 concludes the paper with a discussion of the results.

\section{A model of noisy commitments}

Our analysis relies on the peasant-dictator game, as introduced by van Huyck et al. (1995) and on the investment game (Berg et al., 1995). In both games, an investment is highly efficient, but at the risk of being exploited by the opponent. Two players, $A$ and $B$, interact, where $A$ (the peasant) receives an initial endowment $I_{A}$. Player $A$ has the opportunity to send any amount $a I_{A}$ for $0 \leq a \leq 1$ to player $B$. The amount sent is tripled before it reaches $B$ (the dictator). Player $B$ decides to return any fraction $b$ of the amount for $0 \leq b \leq 1$. Thus, player $B$ returns the amount $b 3 a I_{A}$ to $A$ and keeps $(1-b) 3 a I_{A}$ for himself. Consequently, player $B$ earns $(1-b) 3 a I_{A}$, while $A$ earns the returned amount plus what he did not send (i.e., $\left.(1-a+3 a b) I_{A}\right)$. The entire mechanism of the game is known to both players. Of course, the rational behavior of $B$ implies $b=0$. Since $A$ anticipates this, the subgame perfect equilibrium of the peasant-dictator game/investment game is $a=0$ and $b=0$. No investment occurs. In order to facilitate investments, van Huyck et al. (1995) modify the game, adding a commitment stage. As a result, the peasant-dictator game with a commitment mechanism consists of three consecutive stages. In $t=1$, player $B$ has to commit to a return rate $b$. In $t=2$, the commitment is transferred to $A$. Thus $A$ can condition her share $a$ on the return rate $b$ indicated by player $B$. Finally, in $t=3$, payoffs are realized.

Proposition 1: The weak Nash equilibrium of the peasant-dictator game with a commitment mechanism is $a=1$ and $b=\frac{1}{3}$.

Proof: see Appendix.

However, the commitment is conveyed through a noisy transmission mechanism; this implies that the true commitment is not necessarily 
conveyed. $^{2}$ Denoting the received signal by $s_{b a}$, we introduce a noise mechanism as

$$
s_{b a}= \begin{cases}b & \text { with probability } \frac{1}{2} \\ \min \{1, b+\beta\} & \text { with probability } \frac{1}{2}\end{cases}
$$

for a commonly known $\beta$. Particularly for the following, we restrict the degree of potential contamination to $\beta \in\left(\frac{1}{3}, 1\right]$. We consider only substantial noise, i.e., noise that even contaminates the commitment of $b=0$ such that it appears acceptable. Thus, there is no "obvious" range of unacceptable commitments from the outset. Furthermore, we want to emphasize that this mechanism implies only positively biased signals, i.e., signals that are potentially higher than the true value of $b .^{3}$ One can think of restaurant recommendations that potentially overestimate the quality of meals. Therefore, the peasant-dictator game with a noisy commitment mechanism consists of four consecutive stages. In $t=0$, nature first chooses $s_{b a}$ according to equation (1). In $t=1$, player $B$, who does not know nature's move, commits herself to return a fraction $b$. In $t=2$, player $A$ receives the signal $s_{b a}$ and decides about $a$. Finally, in $t=3$, payoffs are realized. Of course, since we modified the peasantdictator game such that players face a dynamic game with incomplete information, for optimal behavior it is important to consider players' beliefs. Potentially, player $B$ can abuse the noisy commitment mechanism in such a way that she commits herself to return less to player $A$ than player $A$ invested. However, the theoretical analysis yields a different result.

Proposition 2: Sequential rational belief formation in the peasantdictator game with a noisy commitment mechanism yields the following for any pair of commitments $\underline{b}$ and $\bar{b}$, such that $\underline{b} \in\left[0, \frac{1}{3}\right)$ and $\bar{b}=\underline{b}+\beta$ : a) for $\underline{b} \leq 1-2 \beta$, the choice of $\bar{b}$ weakly dominates $\underline{b}$. Therefore, player $A$, who receives a signal $s_{b a} \in[\beta, 1-\beta]$, chooses $a=1$ with probability 1 .

b) for $\underline{b}>1-2 \beta$, the choice of $\underline{b}$ dominates $\bar{b}$ if $\Lambda>\frac{1-\underline{b}-\beta}{\beta}$, while $\bar{b}$ weakly dominates $\underline{b}$ if $\Lambda \leq \frac{1-\underline{b}-\beta}{\beta}$, where $\Lambda$ denotes player $B$ 's belief that player

\footnotetext{
${ }^{2}$ Of course, committing oneself to a future action also creates a strategic advantage (Schelling, 1960) such that the committing party receives all the surplus. Bagwell (1995) shows that even a low level of stochastic noise eliminates this advantage. An experimental analysis of Bagwell's suggestion shows that Bagwell's result holds for the first round, but, as experience increases, the first-mover advantage emerges even in the presence of noise (Huck \& Müller, 2000). Note that we assume a noise mechanism such that the first-mover advantage survives noisy transmission.

${ }^{3}$ It is possible to consider other noise mechanisms that also allow for the uniform or normal distribution of noise; these were analyzed in Bagwell (1995).
} 
A will choose $a>0$, responding to a signal $s_{b a}=\underline{b}+\beta=\bar{b}$. Therefore, player $A$, who receives a signal $s_{b a} \in\left(1-\beta, \frac{1}{3}+\beta\right)$, will choose $a=1$ with probability $\frac{1-\underline{b}-\beta}{\beta}$, and choose $a=0$ with probability $\frac{\underline{b}+2 \beta-1}{\beta}$.

Proof: see Appendix.

With respect to observation 2 , we can conclude that it does not pay for player $B$ to abuse the noisy commitment mechanism. However, for a certain range of commitments, player $A$ will invest $a=1$ only with a probability smaller than one. In the framework of our example, it does not pay for restaurant owners - who know that tourist guides potentially overestimate meal quality - to have low quality meals and speculate on a noisy transmission of commitments. Only if restaurant owners believe that tourists are very likely to follow the guide's recommendation, and do not decrease the probability of following recommendations for high quality restaurants, is it profitable to imitate an excellent restaurant. Yet, the further theoretical analysis shows that abusing the noisy commitment mechanism is not an optimal strategy.

Proposition 3: The weak Nash equilibrium of the peasant-dictator game with a noisy commitment mechanism is $a=1$ and $b=\frac{1}{3} .4$

Proof: see Appendix.

Consequently, the equilibrium prediction remains unchanged for the peasant-dictator game with the commitment mechanism and the peasantdictator game with the noisy commitment mechanism. ${ }^{5}$ Thus it is profitable for restaurant owners to choose to offer reasonable restaurant quality rather than to speculate on a noisy transmission of commitments. As a consequence, the game with the noisy commitment mechanism enables us to observe the "pure" behavioral response of players to a noisy transmission mechanism.

\section{$3 \quad$ Hypotheses}

For laboratory experiments, we chose two degrees of noise as treatment conditions, $\beta=0$ (no-noise) and $\beta=0.4$ (noise). We refer to trustworthy commitments as commitments $b \geq 0.34$ of player $B$, i.e., player $B$ returns at least the amount of money she has received from player $A$, while commitments $b<0.34$ are defined as untrustworthy. Consequently, we refer to player $B$ as the trustee. Likewise, we refer to player $A$ as the trustor. We define trust as a situation in which the trustor chooses $a=1$ as a response to a signal $s_{b a} \in(0.6,0.73]$. Please note that we refer to signals within this interval as problematic since, by proposition 2, this interval characterizes the signals where trustors have to

\footnotetext{
${ }^{4}$ Of course, we have to stress that this result holds only for $\beta \in\left(\frac{1}{3}, 1\right]$.

${ }^{5}$ The first-mover advantage survives the introduction of a noisy commitment mechanism.
} 
choose $a=1$ with a probability smaller than 1 in order to preserve the dominance of trustworthy commitments. Particularly, the probability to invest decreases from 0.975 for $s_{b a}=0.61$ to 0.675 for $s_{b a}=0.73$. Hence, for the noise condition, we find four levels of signals, one untrustworthy, one problematic, and two trustworthy levels, whereas the no-noise condition creates signals at an untrustworthy and a trustworthy level. Figure 1 shows the boundaries of these levels.
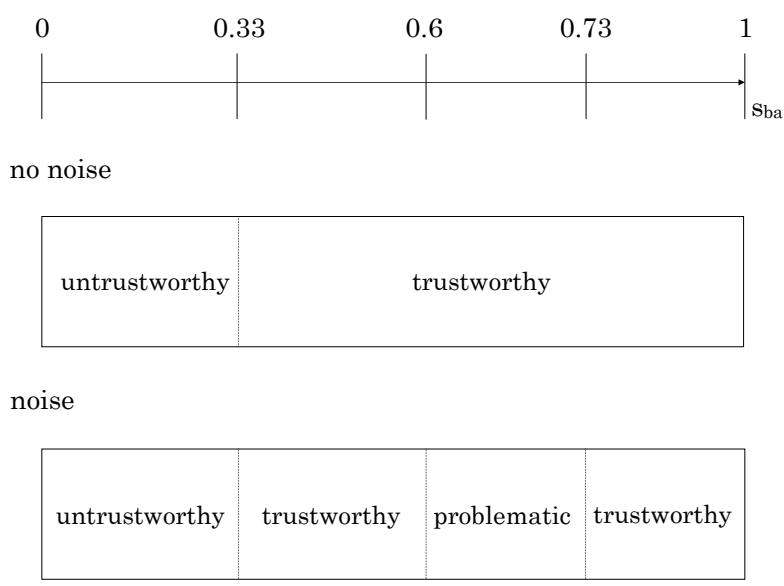

Figure 1: Accuracy of signals

Of course, theory predicts that trustors will choose $a=0$ for untrustworthy signals, while $a=1$ for trustworthy signals. Moreover, given proposition 2, for problematic signals we expect that they will choose $a=1$ with some probability smaller than 1 . As a consequence, trustees will choose the smallest commitment among the trustworthy commitments. Thus, applying proposition 1 to the no-noise condition and proposition 3 to the noise condition, we assume that noisy signals will not change the equilibrium path of the game. The strategic analysis of both treatment conditions predicts that trustees will choose $b=0.34$ and trustors will respond by investing $a=1$ for both conditions. Therefore, for both conditions, investment occurs in such a manner that we can state the rational hypothesis

$H_{\text {ratio: }}$ There is no difference in the efficiency of production between the noise condition and the no-noise condition.

However, the theoretical solution to the game relies on strong assumptions concerning individual rationality. One can easily think of other scenarios that relax this strong precondition. Reconsidering the peasant-dictator game with the commitment mechanism, we find some 
similarities to ultimatum bargaining (Güth et al., 1982), i.e., trustees offer a share of a pie, which trustors accept or reject. Thus, it seems plausible to assume that fairness considerations influence trustees' and trustors' decisions. However, at least there are two relevant fairness norms applicable here. One could ask for an equal split of the production or for an equal split of the surplus of production. It has been well documented that multiple possible foci for fairness judgements result in a self-serving bias (Babcock et al., 1996), i.e., subjects select a fairness standard which favors themselves. Therefore, it seems plausible to assume that trustees would commit themselves to an equal split of the pie, i.e., $b=0.5$, while trustors would expect an equal split of the production surplus, i.e., $b=0.66$, to be fair. Thus, under the no-noise condition, trustors will choose $a=1$ if $s_{b a} \geq 0.66$. Lower signals trigger conflicts between trustors and trustees about the fair division of payoffs, and, as a consequence, may cause efficiency losses. However, for the noise condition, we argue that the acceptance of the equal split of the pie is increased. Earlier studies have shown that the set of alternatives crucially influences fairness norms (e.g., Falk, Fehr \& Fischbacher, 2003). Notice that the commitment suggesting the equal split of the surplus potentially leads to a problematic signal. Hence, there is an "obvious" reason for trustees to consider the fairness norm of $b=0.5$. As a result, the acceptance rate of trustors for the equal split of the pie increases. Overall, more investment occurs under the noise condition. For the fairness hypothesis, we predict

$H_{\text {fair: }}$ : Production is more efficient under the noise condition than under the no-noise condition.

In contrast to the first hypothesis, the off equilibrium hypothesis predicts that noise will have a negative effect on efficiency. In particular, noise will not facilitate investments, as suggested by the fairness hypothesis. On the contrary, it predicts that noise will move responses away from the rational equilibrium towards randomness. The reason for this effect is that noise changes the incentives for behavior off the equilibrium path, and these changes are expected to increase confusion. More specifically, we assume that players engage in some kind of reinforcement learning (e.g., Roth \& Erev, 1995) in order to adjust their investments and commitments in the course of the game. Activities which have been chosen successfully in the past are reinforced, i.e., played more frequently than those which have been less successful. Hence, investments are reinforced if commitments are trustworthy; no investments are reinforced if commitments are untrustworthy. However, both untrustworthy and trustworthy commitments are reinforced if investments occur. Under the no-noise condition, untrustworthy signals are certainly 
detected and not reinforced since trustors choose $a=0$; by contrast investments for trustworthy signals are reinforced. Consequently, behavior converges to the efficient outcome. However, if commitments are noisily transferred under the noise condition, untrustworthy commitments are potentially reinforced by investments. Therefore, it is harder for trustors and trustees to differentiate between the expected values for the different signals. This confusion will lead to less investment, and thus behavior will shift away from the efficient equilibrium. Correspondingly, the off-equilibrium hypothesis predicts that noisy commitments will reduce investments in the long run.

$H_{\text {off }}$ : In the course of the experiment, the efficiency of production decreases under the noise condition, while efficiency increases under the no-noise condition.

Next, we elaborate on the method used to examine the specified predictions and the results of this examination.

\section{Experimental design and results}

The experiment took place at the computer lab at the Israel Institute of Technology (Technion), Haifa, in May 2005. For experiments, we used zTree software (Fischbacher, 1999). Participants were either of type $A$ or $B$ for the entire experiment, but played anonymously in each round with a new partner of the alternative type. As an initial endowment, we choose $I_{A}=12$ Israeli Sheqels (about 2.3 US dollars). In addition, both players received a show up fee of 10 Sheqels. In total, 64 subjects - mostly undergraduate students in their first or second year - participated. Before the experiment began, participants were asked to answer a short questionnaire to ensure that they understood the instructions. Participants needed approximately 25 minutes to play six repeated peasant-dictator games with a commitment mechanism, where the noise parameter was constant for the entire sequence of the experiment. However, only one randomly determined period was payoff relevant. Participants received an average payment of 22 Sheqels (with the payment ranging from 12 to 38 Sheqels).

Figure 2 offers a general overview of commitments $b$ throughout the experiment. As one can see, under the noise condition, the median commitment decreases in the course of the experiment. However, there is a broad range of commitments for both conditions, which remains throughout the course of the experiment. Furthermore, it is obvious that trustees deviate from the game theoretical prediction for the no-noise and the noise condition. Indeed, a statistical analysis of the median $b$ (0.5, with mean absolute difference 0.148 for the no-noise condition, 0.4 , with mean absolute difference 0.148 for the noise condition) indicates 
a significant deviation from the theoretical prediction. ${ }^{6}$ Therefore, we can already reject the rational commitments of trustees. However, we cannot find any significant difference between commitments. ${ }^{7}$
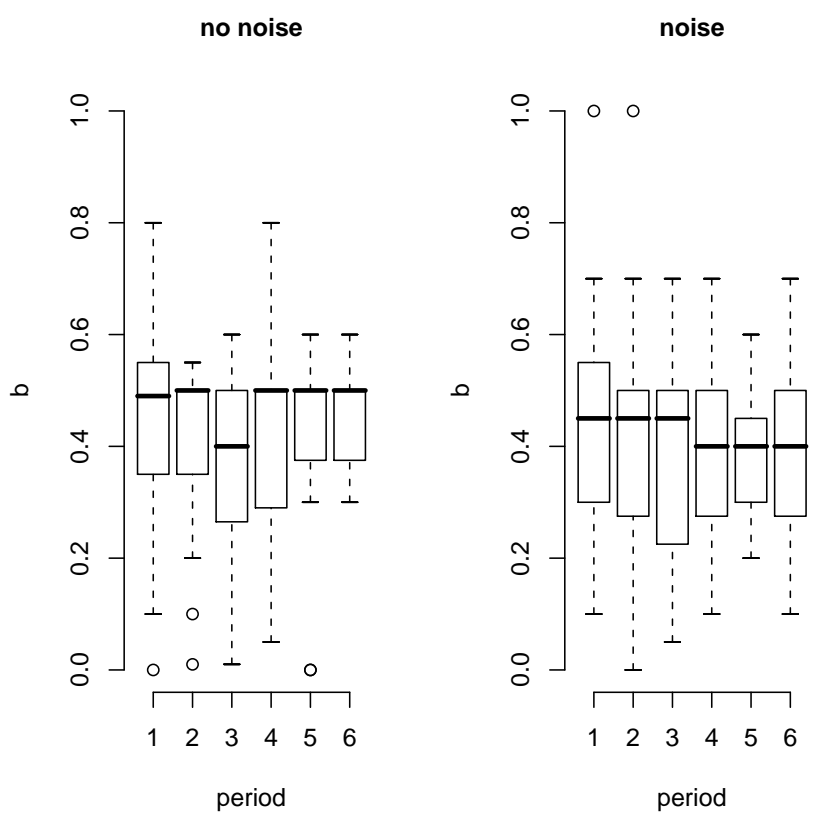

Figure 2: player B commitments by period

Consequently, trustors' behavior also deviates from the equilibrium prediction. Examining the investment $a$, Figure 3 shows the median values (the median absolute deviations) for the different levels of signals. Overall, we find a median a (median absolute deviation of $a$ ) of 1 (0) for the no-noise condition and $0.5(0.74)$ for the noise condition. It seems that trustors rationally invest in the no-noise condition. With respect to rational investment in the noise condition, we find that for $s_{b a}<0.34$ and $s_{b a} \geq 0.74$, trustors indeed behave in accordance with theoretical prediction. Moreover, for these two levels of signals, there is no significant difference in the investments under these conditions. ${ }^{8}$ Thus the data supports the view that trustors invest rationally. However, trustors respond very differently to signals for $0.34 \leq s_{b a}<0.61$ and for $0.61 \leq s_{b a}<0.74$ under differing conditions. Under the noisy

\footnotetext{
${ }^{6} p<0.001$ for both conditions; Wilcoxon signed rank test, two-sided.

${ }^{7} p=0.65$ testing equality; Wilcoxon signed rank test, two-sided.

${ }^{8} p=0.16$; Wilcoxon rank-sum test, two-sided for $s_{b a}<0.34$ and $p=0.397$; Wilcoxon rank-sum test, two-sided for $s_{b a} \geq 0.74$.
} 
condition, they neither choose $a=1$ for $0.34 \leq s_{b a}<0.61$ nor do they $\operatorname{mix} a=0$ and $a=1$ for $0.61 \leq s_{b a}<0.74$. Rather, trustors invest only some proportion of their endowment in response to such commitments. This finding is a first indicator of the trustors' confusion under the noise treatment. Another indicator is the increased variance in trustors' responses to noisy commitments compared to their responses under the no-noise condition. These observations confirm with the off-equilibrium hypothesis. It seems that players are confused by the introduction of noisy commitments and act more randomly.

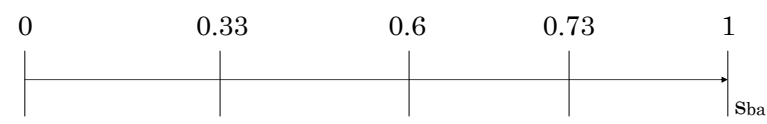

no noise

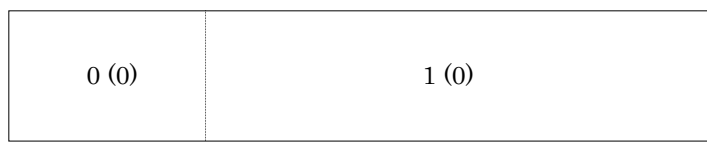

noise

\begin{tabular}{|l|l|l|l|}
\hline & & & \\
& & & \\
& $0.33(0.309)$ & $0.83(0.247)$ & $1(0)$ \\
\hline
\end{tabular}

Figure 3: Median a (median absolute deviation of $a$ ) for different signals

For a more detailed analysis of the response of investments on signals, we define the following dummy variables. Let

$$
\delta_{t r}=\left\{\begin{array}{ll}
1 & \text { if } s_{b a} \text { is trustworthy, } \\
0 & \text { otherwise }
\end{array} \text { and } \delta_{\text {time }}= \begin{cases}1 & \text { if period }>4 \\
0 & \text { otherwise }\end{cases}\right.
$$

Further, we characterize

$$
\delta_{e q 1}=\left\{\begin{array}{ll}
1 & \text { if } s_{b a} \geq 0.5 \\
0 & \text { otherwise }
\end{array} \text { and } \delta_{e q 2}= \begin{cases}1 & \text { if } s_{b a} \geq 0.66 \\
0 & \text { otherwise }\end{cases}\right.
$$

Finally, for data from the noise condition, we define

$$
\delta_{\text {prob }}= \begin{cases}1 & \text { if } s_{b a} \text { is problematic } \\ 0 & \text { otherwise }\end{cases}
$$


We estimate the investment level $a$ as the dependent variable in the simple linear regression model. For data from the no-noise condition, we test

$$
a=x_{0}+x_{1} \delta_{t r}+x_{2} \delta_{e q 1}+x_{3} \delta_{e q 2}+x_{5} \delta_{t r} \times \delta_{\text {time }},
$$

while for data from the noise condition, we test

$$
a=x_{0}+x_{1} \delta_{t r}+x_{2} \delta_{e q 1}+x_{4} \delta_{\text {prob }}+x_{5} \delta_{t r} \times \delta_{\text {time }}+x_{6} \delta_{\text {prob }} \times \delta_{\text {time }} .
$$

For both models, the absolute term $x_{0}$ characterizes the unconditional investment, even for untrustworthy signals. Of course, all theories predict $x_{0}=0$, yet purely altruistic reasons render $x_{0}>0$ (e.g., Forsythe et al, 1994).

According to the rational hypothesis, for equation (3), we predict a coefficient $x_{1}=1$, while $x_{2}, x_{3}$, and $x_{5}$ have no significant influence. To the contrary, if (at least) some fairness concerns matter for trustors, for equation (3), we should find that coefficients $x_{2} \leq 1, x_{3}=1$, and $x_{1}$ and $x_{5}$ have no significance. The off-equilibrium hypothesis predicts that an increase in investments will correspond with trustworthy signals in the course of the experiment since trustworthy commitments are reinforced. Therefore, we expect more investment as a response to trustworthy signals in late periods, i.e., $\delta_{\text {time }}=1$, than in early periods of the experiment. Consequently, we predict $x_{1}=1, x_{5}>0$, and that $x_{2}$ and $x_{3}$ will be insignificant.

For the estimation of equation (4), the rational hypothesis predicts coefficients $x_{1}=1$, while $x_{4}<1$ due to the optimal mix between investments and no investments in response to problematic signals. Other coefficients have no significant effects. Again, if fairness concerns matter for investment decisions, we find the coefficients $x_{2}=1$, and there are no significant effects for the other coefficients. In accordance with the offequilibrium hypothesis, it is expected that untrustworthy commitments will be reinforced. Whereas trustworthy and problematic signals are only rewarded by some investment, i.e., $x_{1}<1$ and $x_{4}<1$, less investment is observed as a response to both problematic and trustworthy signals at the end of the experiment, i.e., $x_{5}<0$ and $x_{6}<0$. Finally, other coefficients have no significant effects. Table 1 shows the results for the estimation of equation (3) and (4). ${ }^{9}$

The results for the no-noise model support the off-equilibrium hypothesis. Confirming earlier experimental results by van Huyck et al. (1995),

\footnotetext{
${ }^{9}$ Standard errors in parenthesis; ${ }^{* * *}$ significant on a $\alpha=0.001$ level, ${ }^{* *}$ significant on a $\alpha=0.01$ level, ${ }^{*}$ significant on a $\alpha=0.05$ level. Joined F-tests for both models reject the hypothesis of insignificant coefficients on an $\alpha=0.001$ level.
} 


\begin{tabular}{ccc} 
& no-noise & noise \\
\hline$x_{0}$ & 0.03 & $0.15^{*}$ \\
& $(0.04)$ & $(0.07)$ \\
$x_{1}$ & $0.83^{* * *}$ & $0.28^{*}$ \\
& $(0.06)$ & $(0.12)$ \\
$x_{2}$ & -0.03 & $0.30^{* * *}$ \\
& $(0.05)$ & $(0.1)$ \\
$x_{3}$ & -0.21 & \\
& $(0.14)$ & \\
$x_{4}$ & & $0.35^{*}$ \\
& & $(0.16)$ \\
$x_{5}$ & $0.10^{*}$ & -0.06 \\
& $(0.05)$ & $(0.09)$ \\
$x_{6}$ & & $-0.73^{*}$ \\
& & $(0.35)$ \\
\hline adj rsquare & 0.77 & 0.34 \\
\hline
\end{tabular}

Table 1: Regression results for equation (3) and (4)

we observe that commitments facilitate investments. Indeed, commitments are positively reinforced. Investments for trustworthy signals increase in the course of the experiment. To the contrary, the results under the noise condition indicate little structured investment. Quite astonishingly, here even trustworthy signals are only partially reinforced. Hence, the mere existence of a noisy transmission mechanism shifts behavior away from the equilibrium and lowers investments. Yet, even untrustworthy signals yield some positive payoffs. Clearly, the results contradict the rational hypothesis, as well as the fairness hypothesis. As predicted by the off-equilibrium hypothesis, random responses arise from experience with experiments. It seems that trustors invest less in order to avoid reinforcing untrustworthy commitments. However, even the cautious investments of trustors are sufficient to reward those commitments. Figure 4 shows the experimentally observed average earnings for trustees, based on submitted commitments. ${ }^{10}$ As one can see, rewards for commitments under the noise condition do not punish untrustworthy commitments. Consequently, a broad range of commitments is reinforced.

Another interesting finding is that the majority of trustees committed to share the production equally, although theory predicts a first-mover

\footnotetext{
${ }^{10}$ Earnings are clustered such that commitments on the ordinal axis represent the midpoint of the class.
} 


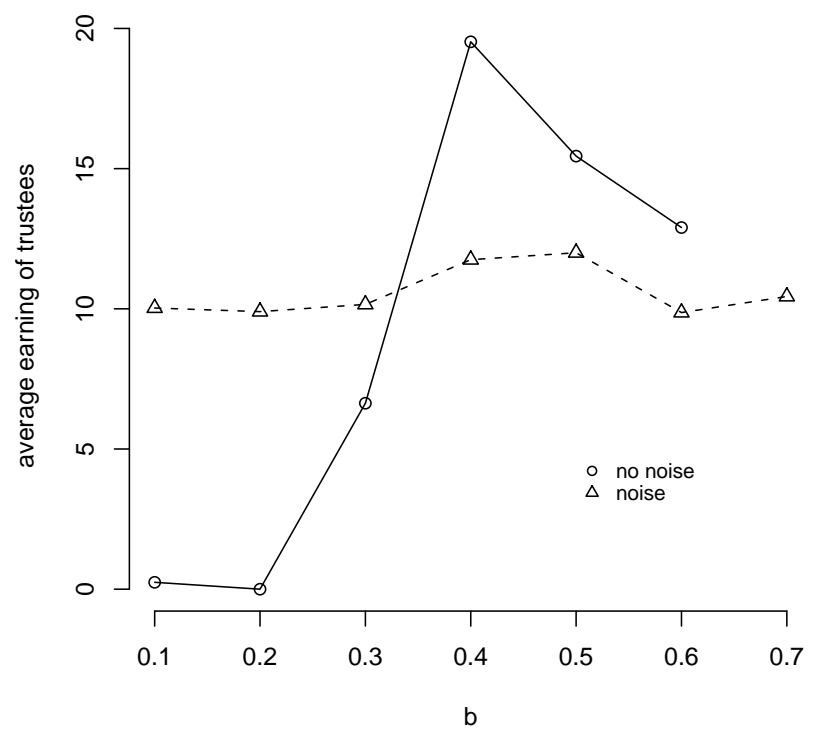

Figure 4: Average earnings of trustees

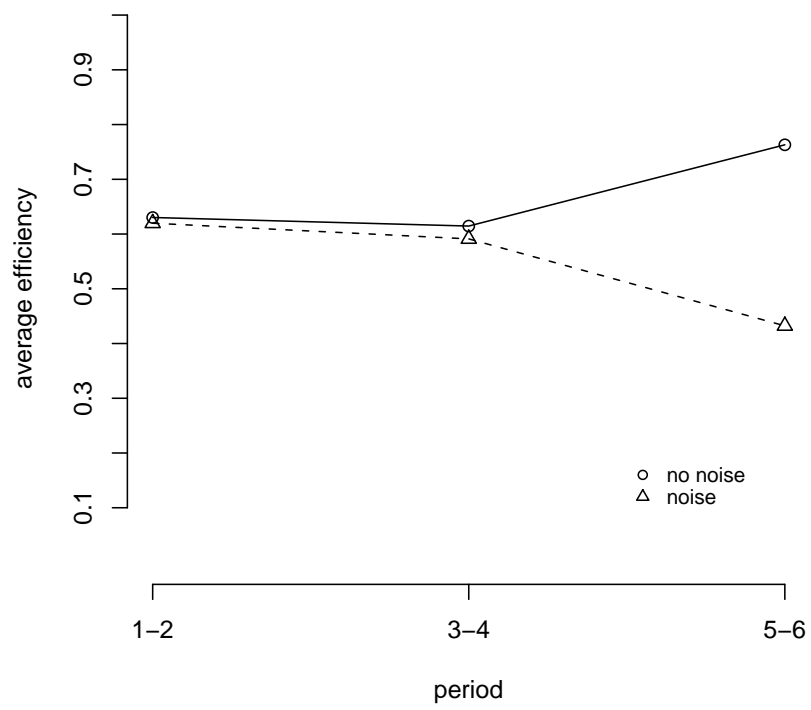

Figure 5: Efficiency in the course of the experiment 
advantage under both conditions. One can assume that trustees' own fairness concerns, as well as the anticipated fairness concerns of trustors, lead to this behavior. Yet trustors responded quite differently to the different treatment conditions. While they responded rationally under the no-noise condition, their response under the noise condition appeared to be more random. It seems that the mere introduction of a potential noisy mechanism leads to a generally lower average investment level, and to a higher variability in trustors' responses. However, these responses reinforce untrustworthy commitments. As a consequence, trustors learn to decrease investments even further in the course of the experiment. Consequently, investment efficiency develops as shown in the next figure. Figure 5 posits efficiency as a function of experience under the noise and no-noise conditions. Since trustworthy signals are also only partially rewarded when there are noisy commitments, in early periods of the experiment the different conditions do not result in different levels of efficiency. This result seems to support $H_{\text {ratio }}$ that both commitment mechanisms facilitate efficiency. With experience, however, efficiency increases under the no-noise condition as a response to reinforced trustworthy commitments, but it decreases under the noise condition due to reinforced untrustworthy behavior. This effect supports $H_{o f f} \cdot{ }^{11}$

\section{Conclusion}

Previous studies on commitments showed that accurate commitments promote efficiency, both theoretically and experimentally (van Huyck et al., 1995). The current data are consistent with this finding. However, in some situations, one cannot be certain that early commitments will be followed up. By examining the effect of commitments in cases where commitments are not necessarily accurate, the current study extends our understanding of the effect of early commitments on trade. We find that noise has a strong effect on players investments in response to such commitments and consequently on efficiency. With respect to the advantage of committing parties predicted by theory, we can conclude that one's own fairness considerations, as well as the anticipated fairness considerations of one's counterparts, eliminate this strategic advantage. This result is quite surprising since, for the context of the peasant-dictator game with a commitment mechanism, fairness considerations cannot be identified as having an important influence on the parties who have to invest. Rather, we find that peasants respond rationally, which is puzzling, given the results of ultimatum bargaining. One possible explanation is that this difference in behavior may be attributed to the fact that these

\footnotetext{
${ }^{11} p<0.03$ for the linear trend in each group; Mann-Whitney test, one-sided.
} 
games are framed differently.

Yet the differences between efficiency under the two conditions are revealed only after some time. This finding is consistent with earlier findings that games with similar equilibria, but different off-equilibrium incentives, elicit different behavior since players' behavior is reinforced differently. Therefore, players who begin these game away from the equilibrium may learn very different things (Roth \& Erev, 1995). The current study shows that, when commitments are accurate, players learn to interact efficiently. However, when commitments are noisy, investments are lowered as a result of the introduction of a potentially noisy mechanism. Moreover, trustors cannot learn to differentiate between signals; consequently, trustees do not learn to interact efficiently. Apparently, inaccurate information impairs efficiency. Compared with accurate recommendations, tourist guides that rate the qualities of meals too high do not enhance the profits restaurants earn from tourists. Rather, they harm the profits of all restaurants, even cutting into the profits of good quality ones. The current analysis implies that positively biased recommendations may facilitate efficiency only for a short time. However, in the long run such recommendations might impair efficiency.

\section{References}

[1] Andreoni, J., P.M. Brown \& L. Vesterlund (2002), What makes an allocation fair? Some experimental evidence, Games and Economic Behavior 40, 1-24.

[2] Babcock, L., X. Wang \& G. Loewenstein (1996), Choosing the wrong pond: Social comparisons in negotiations that reflect a self-serving bias, The Quarterly Journal of Economics, 111 (1), 1-19.

[3] Bagwell, K. (1995), Commitment and observability in games, Games and Economic Behavior 8, 271-280.

[4] Berg, J., J. Dickhaut, \& K. McCabe (1995), Trust, reciprocity, and social history, Games and Economic Behavior 10, 122-142.

[5] Bolton, G.E., \& A. Ockenfels (2000), ERC: A theory of equity, reciprocity, and competition, American Economic Review 90 (1), 166193.

[6] Bolton, G.E., E. Katok, \& A. Ockenfels (2004), How effective are electronic reputation mechanisms? An experimental investigation, Management Science 50 (11), 1587-1602.

[7] Burnham, T., K. McCabe \& V.L. Smith (2000), Friend-or-foe intentionality priming in an extensive form trust game, Journal of Economic Behavior $\&$ Organization, 43, 57-73. 
[8] Falk, A., E. Fehr \& U. Fischbacher (2003), On the nature of fair behavior, Economic Inquiry 41, 20-26.

[9] Fehr, E. \& K.M. Schmidt (1999), A theory of fairness, competition, and cooperation The Quarterly Journal of Economics 114 (3), 817868.

[10] Fischbacher, U. (1999), z-Tree - Zurich Toolbox for Readymade Economic Experiments - Experimenter's Manual, Working Paper No. 21, Zurich: Institute for Empirical Research in Economics, University of Zurich.

[11] Forsythe, R., J.L. Horowitz, N.E. Savin \& M. Sefton (1994), Fairness in simple bargaining experiments, Games and Economic Bahavior 6, 347-369.

[12] Güth, W., R. Schmittberger \& B. Schwarze (1982), An experimental analysis of ultimatum bargaining, Journal of Economic Behavior $\&$ Organization 3, 367-388.

[13] Hart, O. (1995), Firms, Contracts, and Financial Structure, Oxford: Oxford University Press.

[14] Huck, S. \& W. Müller (2000), Perfect versus imperfect observability - An experimental test of Bagwell's results, Games and Economic Behavior 31, 174-190.

[15] Johnson, E.J., J. Hershey, \& H. Kunreuther (1993), Framing, probability distortions, and insurance decisions, Journal of Risk and Uncertainty 7 (1), 35-51.

[16] Kreps, D. (1990), Corporate culture and economics theory, in: Alt, J., K. Shepsle (eds.) Perspectives on positive political economy, pp. 43-90, Cambridge, Cambridge University Press.

[17] Lucking-Reiley, D., D. Bryan, N. Prasad \& D. Reeves (1999), Pennies from ebay: The determinants of price in online auctions, working paper, Nashville: Vanderbilt University.

[18] Melnik , M.I. \& J. Alm (2002), Does a seller's e-commerce reputation matter? Journal of Industrial Economics 50 (3), 337-349.

[19] Prasnikar, V. \& A.E. Roth (1992), Considerations of fairness and strategy: Experimental data from sequential games, The Quarterly Journal of Economics 107 (3), 865-888.

[20] Rabin, M. (1993), Incorporating fairness into game theory and economics, American Economic Review 83 (4), 1281-1302.

[21] Resnick, P., R. Zeckhauser, J. Swanson \& K. Lockwood (2002), The value of reputation on ebay: A controlled experiment, working paper, Ann Arbor: University of Michigan.

[22] Roth, A.E. \& I. Erev (1995), Learning in extensive-form games: Experimental data and simple dinamic models in the intermediate term, Games and Economic Behavior 8, 164-212. 
[23] Schelling, T. (1960), The strategy of conflict, Cambridge, M.A.: Harvard University Press.

[24] van Huyck, J.B., R.C. Battalio, \& M.F. Walters (1995), Commitment versus discretion in the peasant-dictator game, Games and Economic Behavior 10, 143-170. 


\section{Appendix}

\section{A. Proof of Proposition 1}

Proof Let us denote player $A$ 's (B's) payoff as $\pi_{A}(a)\left(\pi_{B}(b)\right.$, respectively).

Clearly, for every commitment $b<\frac{1}{3}$ that is received by player $A$, a simple comparison of payoffs $\pi_{A}$ for the choices $a=0$ and $\bar{a}>0$ yields $\pi_{A}(0)>\pi_{A}(\bar{a})$, so that $\bar{a}$ is dominated.

For every $b=\frac{1}{3}, \pi_{A}(1)=\pi_{A}(\underline{a})$ for $\underline{a}<1$, so that player $A$ is indifferent about this commitment.

Finally, for every $b>\frac{1}{3}, \pi_{A}(1)>\pi_{A}(\underline{a})$ for $\underline{a}<1$, so that $\underline{a}$ is dominated.

Thus, player $A$ 's best response is full investment if the commitment exceeds $\frac{1}{3}$, and non-investment if the commitment falls below $\frac{1}{3}$. Player $B$ 's decision problem is to choose $b$, which maximizes her payoff. Assuming common knowledge of rationality, a simple comparison of payoffs yields $\pi_{B}(\underline{b})<\pi_{B}(\bar{b})<\pi_{B}\left(b^{\prime}\right)$ for $0 \leq \underline{b}<\frac{1}{3}, \frac{1}{3}<\bar{b} \leq 1$, and $b^{\prime}=\frac{1}{3}$. Of course, $\pi_{B}(\underline{b})=0$ and, defining without any loss of generality $\bar{b}:=\frac{1}{3}+\varepsilon$, $\pi_{B}(\bar{b})=\left(\frac{2}{3}-\varepsilon\right) 3 I_{A}<\frac{2}{3} 3 I_{A}=\pi_{B}\left(b^{\prime}\right)$, based on $A$ 's best responses.

\section{B. Proof of Proposition 2}

Recall that sequential rational belief formation requires, for both players $A$ and $B$, belief vectors $\widetilde{g}_{a}$ and $\widetilde{g}_{b}$, respectively, which characterize the probabilities for all types of games consistent with their information partitions.

Proof Since noise in our commitment mechanism is only a potential positive deviation, $\widetilde{g}_{a}\left(b<\frac{1}{3} \mid s_{b a}<\frac{1}{3}\right)=1$. Also $\widetilde{g}_{a}\left(b<s_{b a} \mid s_{b a}<\beta\right)=0$, while $\widetilde{g}_{a}\left(b=s_{b a} \mid s_{b a}<\beta\right)=1$. Thus, referring to proposition 1 , A's best responds to $s_{b a}<\frac{1}{3}$ by choosing $a=0$, and she best respond to $s_{b a}=s_{b a}^{\prime}$ such that $\frac{1}{3} \leq s_{b a}^{\prime}<\beta$ if she chooses $a=1$. Likewise, $\widetilde{g}_{a}\left(b<\frac{1}{3} \mid s_{b a}>\frac{1}{3}+\beta\right)=0$, while $\widetilde{g}_{a}\left(b>\frac{1}{3} \mid s_{b a}>\frac{1}{3}+\beta\right)=1$. Consequently, $A$ chooses $a=1$ for $s_{b a}>\frac{1}{3}+\beta$. Therefore, $\widetilde{g}_{b}\left(a=1 \mid b=b^{\prime}\right)=1$ for $b^{\prime} \geq \frac{1}{3}+\beta$, and $\widetilde{g}_{b}\left(a=1 \mid b=b^{\prime \prime}\right)=1$ for $\frac{1}{3} \leq b^{\prime \prime}<\beta$.

For any $b=\underline{b}$ in $0 \leq \underline{b}<\frac{1}{3}$, player $B$ entertains $\widetilde{g}_{b}\left(a=1 \mid b=\underline{b}, s_{b a}=\right.$ $\left.s_{b a}^{\prime}\right):=\Lambda^{\prime}$, where $s_{b a}^{\prime}=\underline{b}+\beta$. To the contrary, $\widetilde{g}_{b}\left(a=1 \mid b=\underline{b}, s_{b a}=\underline{b}\right)=$ 0 . For any $b=\bar{b}$ in $\beta \leq \bar{b}<\frac{1}{3}+\beta$, player $B$ entertains $\widetilde{g}_{b}(a=1 \mid b=$ $\left.\bar{b}, s_{b a}=\bar{b}\right):=\Lambda^{\prime \prime}$. To the contrary, $\widetilde{g}_{b}\left(a=1 \mid b=\bar{b}, s_{b a}=\bar{b}+\beta\right)=1$. Notice that $\Lambda:=\Lambda^{\prime}=\Lambda^{\prime \prime}$ for $\underline{b}+\beta=\bar{b}$ since player $A$ cannot differentiate among the two cases.

A comparison of expected payoffs of player $B$ shows that $E(\pi(\bar{b}))$ dominates $E(\pi(\underline{b}))$ if $1-\underline{b}-\beta-\beta \Lambda<0$, thus, if $\underline{b} \leq 1-2 \beta E(\pi(\bar{b}))$ dominates $E(\pi(\underline{b}))$ for all $\Lambda \in[0,1]$. Since $E\left(\pi_{A}(1 \mid \bar{b})\right)>E\left(\pi_{A}(0 \mid \bar{b})\right) \forall \bar{b}$, 
player $A$, receiving $s_{b a}^{\prime}$ for $\beta \leq s_{b a}^{\prime} \leq 1-\beta$, optimally chooses $a=1$ with probability one.

However, for $\underline{b}>1-2 \beta$ for $\Lambda>\frac{1-\underline{b}-\beta}{\beta}$, one finds that $E\left(\pi_{B}(\underline{b})\right)$ dominates $E\left(\pi_{B}(\bar{b})\right)$, while $E\left(\pi_{B}(\bar{b})\right)$ dominates $E\left(\pi_{B}(\underline{b})\right)$ for $\Lambda \leq \frac{1-\underline{b}-\beta}{\beta}$. Since $E\left(\pi_{A}(\bar{b})\right)>E\left(\pi_{A}(\underline{b})\right) \forall \bar{b}$, player $A$, receiving $s_{b a}^{\prime \prime}$ for $1-\beta<s_{b a}^{\prime \prime}<$ $\frac{1}{3}+\beta$, optimally chooses $a=1$ with probability $\frac{1-\underline{b}-\beta}{\beta}$ and $a=0$ with probability $\frac{2 \beta+\underline{b}-1}{\beta}$.

\section{Proof of Proposition 3}

Proof Referring to proposition 2, we know that $A$ best responds to $s_{b a}=s_{b a}^{\prime}$ such that $\frac{1}{3} \leq s_{b a}^{\prime}<\beta$ by choosing $a=1$; it follows with respect to proposition $1 \arg \max _{b^{\prime \prime}} E\left(\pi_{B}\left(b^{\prime \prime}\right)\right)=\frac{1}{3}$. Hence, simple calculations of expected payoffs yields $E\left(\pi_{B}\left(b^{\prime}\right)\right)=\left(1-b^{\prime}\right) 3 I_{A}<2 I_{A}=E\left(\pi_{B}\left(\frac{1}{3}\right)\right)$ for $b^{\prime} \geq \frac{1}{3}+\beta$. Likewise, $E\left(\pi_{B}(\bar{b})\right)=3 I_{A}\left[\frac{1}{2} \Lambda(1-\bar{b})+\frac{1}{2}(1-\bar{b})\right]<2 I_{A}$ for $\beta \leq \bar{b}<\frac{1}{3}+\beta$. Finally, $E\left(\pi_{B}(\underline{b})\right)=\frac{1}{2} \Lambda(1-\underline{b}) 3 I_{A}<2 I_{A}$ for $0 \leq \underline{b}<\frac{1}{3}$.

\section{Instructions for player $\mathrm{A}^{12}$}

In this experiment, you will play six different games against different players. In all the games, you will be player A. Each game involves two players. For each game, you'll be endowed with 12 Sheqels. You are asked to decide how much of this amount you will transfer to player B and how much you will keep for yourself. You may transfer any integer between 0 (transfer nothing) and 12 (transfer the whole amount). The amount transferred is multiplied by 3 . Then, you will get back the amount that $\mathrm{B}$ decides to return. To make your decision easier, player B will send you a signal at the beginning of the game with the proportion that he commits to return from the amount that you will send. The amount that B returns will be the committed proportion from the multiplied transferred amount.

The signal that you'll receive is not always accurate. There is a $50 \%$ chance that the signal will be the proportion that $\mathrm{B}$ has committed to return. However, there is a $50 \%$ chance that the signal will be the true proportion, plus 0.4. Signals that exceed 1 will be presented as signals of $1 .{ }^{13}$

Your earnings will be the amount that you keep for yourself, plus the amount that B sends back. B's earnings will be the amount that he keeps for himself. At the end of the experiment (after the six games), one game will be randomly selected. The payment from this game will be your earnings for the experiment. Good luck.

\footnotetext{
${ }^{12}$ We present the English translation of the instructions for the noise condition; deviations in the no-noise treatment are indicated by footnotes.

${ }^{13}$ This paragraph is missing under the no-noise condition.
} 


\section{E. Instructions for player B}

In this experiment, you will play six different games against different players. In all games, you will be player B. Each game involves two players. In each game, player A receives 12 Sheqels. He is asked to decide how much of that amount to send you. Player $\mathrm{A}$ is allowed to transfer any integer number between 0 (to transfer nothing) and 12 (to transfer the whole amount). The amount transferred is multiplied by 3. At the beginning of the game (before A makes his decision), you are asked to send a commitment to player A about the proportion of the amount sent that you will return to A. You may choose any proportion between 0 (to return nothing) and 1 (to return the whole amount).

The signal that is received by A regarding your commitment is not always accurate. There is a $50 \%$ chance that A will receive a signal that is the true proportion. However, there is $50 \%$ chance that A will receive a signal that is the true proportion, plus 0.4. Signals that exceed 1 will be presented as signals of $1 .^{14}$

The amount that B returns to player A will be the committed proportion from the multiplied transferred amount. Your earnings will be the amount that you receive, minus the amount that you send back to player A. A's earnings will be the amount that he keeps for himself, plus the amount that you return. At the end of the experiment (after the six games), one game will be randomly selected. The payment for this game will be your earnings for the experiment. Good luck.

\footnotetext{
${ }^{14}$ This paragraph is missing under the no-noise condition.
} 